OPEN ACCESS

Edited by:

Peter Sörös,

University of Oldenburg, Germany

Reviewed by:

Tomi Karjalainen,

Turku PET Centre, Finland

Johanna M. Jarcho,

Stony Brook University, United States

*Correspondence:

Eva M. Bauch

eva.bauch@medicalschool-

hamburg.de

Nico Bunzeck

nico.bunzeck@uni-luebeck.de

Received: 05 July 2017 Accepted: 11 December 2017 Published: 21 December 2017

Citation:

Bauch EM, Andreou C, Rausch VH and Bunzeck N (2017) Neural Habituation to Painful Stimuli is Modulated by Dopamine: Evidence from a Pharmacological fMRI Study.

Front. Hum. Neurosci. 11:630. doi: 10.3389/fnhum.2017.00630

\section{Neural Habituation to Painful Stimuli Is Modulated by Dopamine: Evidence from a Pharmacological fMRI Study}

\author{
Eva M. Bauch ${ }^{1,2 *}$, Christina Andreou ${ }^{3}$, Vanessa H. Rausch ${ }^{1,4}$ and Nico Bunzeck ${ }^{1,5 *}$ \\ ${ }^{1}$ Department of Systems Neuroscience, University Medical Center Hamburg-Eppendorf, Hamburg, Germany, ${ }^{2}$ Medical \\ School Hamburg (MSH), University of Applied Science and Medical University, Hamburg, Germany, ${ }^{3}$ Center for Gender \\ Research and Early Detection, University Psychiatric Clinics Basel, Basel, Switzerland, ${ }^{4}$ Department of Diagnostic and \\ Interventional Radiology and Nuclear Medicine, University Medical Center Hamburg-Eppendorf, Hamburg, Germany, \\ ${ }^{5}$ Institute of Psychology I, University of Lübeck, Lübeck, Germany
}

In constantly changing environments, it is crucial to adaptively respond to threatening events. In particular, painful stimuli are not only processed in terms of their absolute intensity, but also with respect to their context. While contextual pain processing can simply entail the repeated processing of information (i.e., habituation), it can, in a more complex form, be expressed through predictions of magnitude before the delivery of nociceptive information (i.e., adaptive coding). Here, we investigated the brain regions involved in the adaptation to nociceptive electrical stimulation as well as their link to dopaminergic neurotransmission (placebo/haloperidol). The main finding is that haloperidol changed the habituation to the absolute pain intensity over time. More precisely, in the placebo condition, activity in left postcentral gyrus and midcingulate cortex increased linearly with pain intensity only in the beginning of the experiment and subsequently habituated. In contrast, when the dopaminergic system was blocked by haloperidol, a linear increase with pain intensity was present throughout the entire experiment. Finally, there were no adaptive coding effects in any brain regions. Together, our findings provide novel insights into the nature of pain processing by suggesting that dopaminergic neurotransmission plays a specific role for the habituation to painful stimuli over time.

Keywords: pharmacological fMRI, EEG, pain, habituation, haloperidol

\section{INTRODUCTION}

The accurate perception of nociceptive information, such as an electric shock, is crucial for survival and depends on the absolute intensity of the stimulus. For instance, increasing the magnitude of aversive stimulation leads to increased pain perception as well as increased neural activity in pain responsive brain regions, including somatosensory cortices, insular cortex and mid/anterior cingulate cortex (ACC; for a review see Iannetti and Mouraux, 2010). Although such absolute coding has frequently been reported, the processing of aversive information also depends on contextual factors. Specifically, neural responses to nociceptive stimuli decrease as a function of repetition. This is known as habituation (Glaser and Whittow, 1953). This habituation effect in response to electro-dermal stimulation was observed in the above-mentioned pain associated brain regions over consecutive experiment blocks, as shown with fMRI (Bingel et al., 2007; Mobascher et al., 2010) and simultaneous EEG-fMRI (Christmann et al., 2007). Similarly, in an fMRI study, 
Nickel et al. (2014) observed habituation to electrical stimuli in the secondary somatosensory cortex, insula, ACC, dorsolateral prefrontal cortex and inferior parietal lobule. In line with these neural effects, repeated exposure of the same painful stimulus, including heat, leads to decreased pain ratings (May et al., 2012).

While neural habituation to pain appears to be a protective mechanism in healthy humans, dysfunctional habituation may play a role in the chronification of pain (e.g., Bingel et al., 2007; Rodriguez-Raecke et al., 2014). Indeed, studies in chronic pain patients, for instance with chronic low back pain, migraine or fibromyalgia, showed attenuated habituation to pain when compared to healthy controls (e.g., Peters et al., 1989; Schoenen et al., 1995; de Tommaso et al., 2011). Importantly, several chronic pain pathologies such as fibromyalgia or burning mouth syndrome have been associated with dopaminergic deficits (e.g., Brefel-Courbon et al., 2005; Wood et al., 2007; Potvin et al., 2009; de Tommaso et al., 2011; Jarcho et al., 2012) indicating a link between habituation to pain and dopaminergic neurotransmission. For instance, patients with Parkinson's disease, which is mainly characterized by a deficit of dopamine, showed attenuated habituation to nociceptive stimulation while they were off medication, while habituation was evident when dopamine was increased by levodopa treatment (dopamine precursor; Brefel-Courbon et al., 2005; Schestatsky et al., 2007). Moreover, formalin-induced nociception can be enhanced through the injection of D2 antagonists into the dorsolateral striatum (Magnusson and Fisher, 2000), and the dopaminergic substantia nigra/ventral tegmental area (SN/VTA) responds to painful stimuli as a function of probability (e.g., in animals: Matsumoto and Hikosaka, 2009; Bromberg-Martin et al., 2010; in humans: Bauch et al., 2014; Pauli et al., 2015) further suggesting a role of dopamine in pain processing.

Apart from habituation, contextual predictions also modulate pain processing. For instance, in direct comparison a high intensity electrical stimulation is perceived as more painful than a low intensity electrical stimulation (i.e., absolute coding); however, when high intensity stimulation is expected but the low one is delivered, it may be perceived as less intense. At the neural level, this can be related to neural adaptation which is a general property of neurons (Ohzawa et al., 1982; Brenner et al., 2000; Fairhall et al., 2001; Brown et al., 2008), and, more specifically, to the prediction error mechanism, which quantifies the difference between expected and received outcomes. Importantly, prediction errors adaptively scale according to the expected range of momentarily possible outcomes, which allows neurons to maintain high sensitivity. This neural mechanisms is well established in the reward literature (see below) and known as “adaptive coding” (e.g., Tobler et al., 2005; Bunzeck et al., 2010; Kobayashi et al., 2010; Park et al., 2012; Diederen et al., 2017).

For instance, in a single cell recording study in monkeys (Tobler et al., 2005), visual cues were followed with equal probability by either a small or medium reward (i.e., outcome), and another cue was associated with an upcoming large or medium reward. In both contexts, the larger of the two possible reward outcomes (i.e., medium and large) increased activity in the SN/VTA, while the relatively smaller reward led to activity decreases (i.e., scaled prediction errors).
Similar forms of adaptive coding have been observed in the human ventral striatum, prefrontal cortex and medial temporal lobe (Tremblay and Schultz, 1999; Nieuwenhuis et al., 2005; Padoa-Schioppa and Assad, 2008; Padoa-Schioppa, 2009; Bunzeck et al., 2010). Moreover, there is evidence that dopamine modulates adaptive coding of appetitive information in the human midbrain and striatum (Diederen et al., 2017), which seem to be functionally connected (Park et al., 2012).

With regard to pain processing, several imaging studies have shown that contextual predictions (similar to adaptive coding) modulate activity in higher order pain-associated brain regions, including the insula and ACC (Wiech and Tracey, 2009; Ploner et al., 2011; Leknes et al., 2013; for a different finding see Winston et al., 2014). In particular, Leknes et al. (2013) presented within a block-design moderately painful heat stimuli either in a context with intense pain (i.e., relief context) or in a context with non-painful warm stimulation (i.e., control context). Both contexts were predicted by a unique cue; as such the moderately painful heat stimulus could be the best outcome (i.e., relief context) or the worst outcome (i.e., control context). In line with the literature on adaptive reward processing, pleasantness ratings were dependent on the context (called "hedonic flip" by the authors), and skin conductance as well as hemodynamic responses in the insula and dorsal anterior cingulate were higher in the control context as compared to the relief context. Although this activity pattern provides initial evidence for adaptive coding, the link to dopaminergic neuromodulation has not been demonstrated.

In sum, there is independent empirical evidence for: (a) absolute and (b) adaptive coding of painful stimuli, and for (c) neural habituation in pain responsive brain regions. However, the relationship to dopaminergic neurotransmission in healthy human subjects remains unclear. Therefore, we used a double-blind within-subject pharmacological fMRI study (placebo/haloperidol: dopamine antagonist) to investigated the role of dopamine in pain processing with a focus on absolute coding, adaptive coding and neural habituation.

To this end, the experiment consisted of two absolute tasks (phase I and phase III), where subjects rated the absolute intensity of an electro-tactile stimulus (low, medium, high) received on the back of the hand. Between these two phases, we used a task in which contextual predictions to a nociceptive event were manipulated across trials (i.e., adaptive task, phase II). Here, volunteers were presented with three different visual cues (i.e., triangle, square, diamond) predicting: (1) a high or medium electro-tactile stimulus; (2) a medium or low electro-tactile stimulus; or (3) a high or medium electro-tactile stimulus. We hypothesized neural habituation as well as adaptive coding in pain-associated areas, including the insula and ACC (Leknes et al., 2013), prefrontal cortex and possibly mesolimbic brain regions (SN/VTA, ventral striatum; Bunzeck et al., 2010; Leknes et al., 2013; Winston et al., 2014; Diederen et al., 2016). Moreover, we expected that neural habituation to absolute pain magnitude and adaptive coding diminishes in dopaminergic and pain-associated brain regions, when blocking dopaminergic D2 receptors with haloperidol. 


\section{MATERIALS AND METHODS}

\section{Participants}

Twenty-six participants took part in the fMRI experiment, but only 20 were included in further analyses (mean age: 25 years; age range: 19-28 years; 13 women) due to technical problems with the scanner or digitimer (electrical stimulation). All participants were healthy, right-handed and had normal or corrected-to-normal vision, without a history of neurological, psychiatric, or medical disorders or any current medical problems. This study was carried out in accordance with the recommendations of "Ethikkomission der Ärtzekammer Hamburg" with written informed consent from all subjects.

\section{Task}

In order to assess dopaminergic effects on pain processing, a randomized double-blind within-subject design (i.e., $1.5 \mathrm{mg}$ haloperidol/placebo) was used. Haloperidol is an established substance to treat psychiatric disorders including schizophrenia. More specifically, haloperidol is a dopaminergic antagonist that blocks mainly D2 receptors (e.g., Kapur et al., 1997). In correspondence with previous research, our dose of haloperidol has been shown to be an acceptable compromise between sufficient D2 receptor inhibition (between 60\% and $80 \%$ ) and minimization of side effects (Kapur et al., 1997).

The entire experiment took place in 3 days. During the first day, participants practiced a condensed version of the experiment to ensure high performance in all tasks, which will be explained in more detail below (see Figure 1). Furthermore, they received information regarding the MRI procedure and pharmacological manipulation. Participants were assigned to receive placebo on day 2 and haloperidol on day 3 or vice versa in a randomized and double-blinded fashion. The time interval between placebo-day and haloperidol-day was at least 7 days long (mean interval: 15 days) to allow a washout of haloperidol.

The experimental procedure was identical on both days of the pharmacological manipulation. Drug intake was scheduled $2.5 \mathrm{~h}$ before the start of the first phase in the MRI scanner, when haloperidol reaches significant plasma concentration (e.g., Andreou et al., 2014), and participants were asked to not eat $2 \mathrm{~h}$ before drug intake. Blood pressure was measured and a questionnaire about their subjective state and possible side effects was completed at three time points: before drug intake, $2.5 \mathrm{~h}$ after drug intake and after the experiment (i.e., approximately

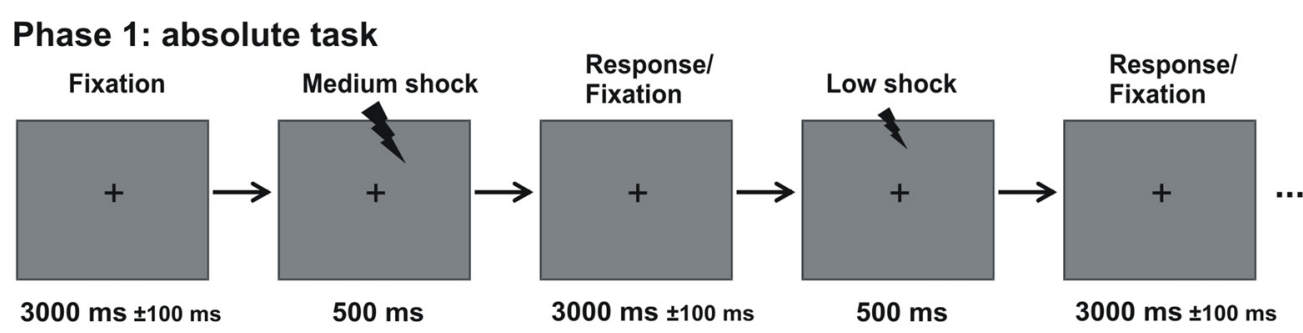

Phase 2: adaptive task

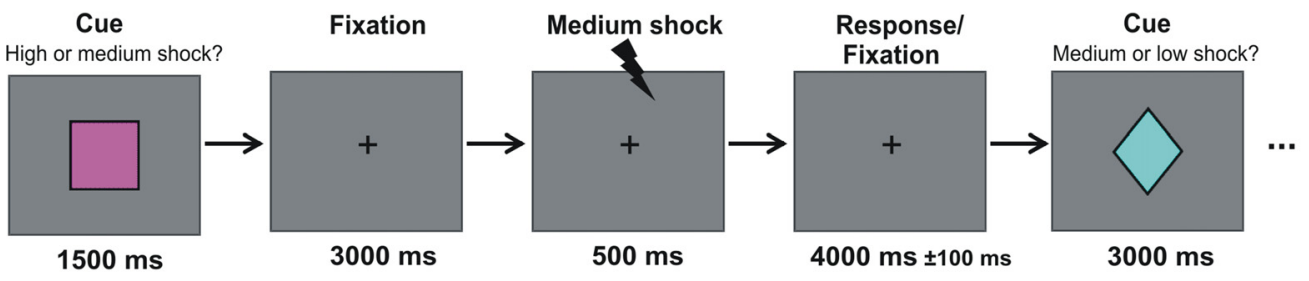

Phase 3: absolute task

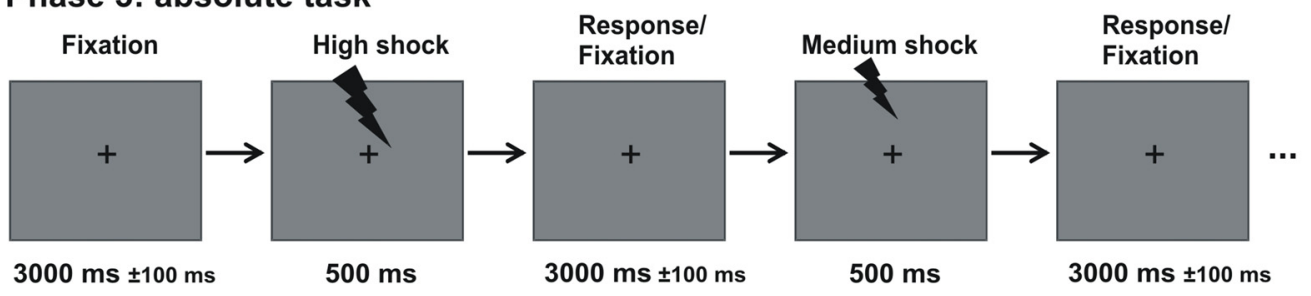

FIGURE 1 | Experimental design. Participants underwent three phases. In the first and third phase (absolute task), electrical stimulation with three different magnitudes were presented randomly intermixed to the back of the hand. Subjects had to make low/medium/high judgments (see experimental procedures for details). In between both phases, participants took part in the adaptive task, where they learned the association between three contexts and three visual cues that predicted with $50 \%$ probability either: (1) a high or a medium stimulation; (2) a medium or a low stimulation; or (3) a high or a low stimulation. Participants made a relative low/high judgment (see experimental procedures for details). 
$4.5 \mathrm{~h}$ after drug intake). After placing the participant into the MRI scanner, an electrode was placed on the back of their right hand, which was followed by calibrating the magnitude for the highest possible electrical stimulation that was applied during the experiment.

Three different stimulation magnitudes were used during the experiment: low, medium and high. The stimulation magnitude was adjusted by varying the number of trains of consecutive electrical pulses of $2 \mathrm{~ms}$ each. Electrical stimulation was applied for $500 \mathrm{~ms}$ in total (i.e., high stimulation: 10 trains of $2 \mathrm{~ms}$ electrical pulses separated by an interval of $50 \mathrm{~ms}$; medium stimulation: six trains of 2 ms pulses separated by an interval of $83.3 \mathrm{~ms}$; low stimulation: four trains of $2 \mathrm{~ms}$ pulses separated by an interval of $125 \mathrm{~ms}$ ). The time window between the first pulse and offset of the last pulse was identical for all shock intensities. This procedure was based on several published studies using the same electro-tactile stimulation system (Haaker et al., 2013; Lonsdorf et al., 2014; Sjouwerman et al., 2015). During calibration, participants were asked to rate the intensity of the electrical stimulation with 10 trains of electrical pulses on a visual analog scale (VAS) ranging from 0 (i.e., electrical stimulation is not perceptible) to 10 (i.e., electrical stimulation is intolerable). An intensity of seven for the electrical stimulation was used as highest possible nociceptive stimulus throughout the experiment. The magnitude of the medium and low stimulation conditions were adjusted accordingly by reducing the number of trains of pulses to six or four, respectively.

The experiment consisted of three consecutive phases: an absolute task (phase 1); an adaptive task (phase 2); and again an absolute task (phase 3; see Figure 1). The visual stimuli were presented via a mirror system attached to the head coil of the scanner. Throughout the first phase (absolute task), a fixation cross was presented in the center of a gray screen. A series of in total 60 consecutive electrical stimulations was applied to the back of the right hand. The three stimulation magnitudes were randomly intermixed (20 trials per condition) and each stimulation was separated by an inter-stimulus interval of $3000 \pm 100 \mathrm{~ms}$. Participants were asked to judge the magnitude of the electrical stimulation (i.e., low, medium, high) by pressing one of three buttons. The contingency between magnitude and button was randomly assigned across participants. Accuracy and speed were stressed. This phase took approximately $5 \mathrm{~min}$ in total. Ten practice trials were shown before the actual task to ensure that participants familiarized themselves with this task.

The second phase included a modified version of an established paradigm used in reward studies investigating adaptive coding (Tobler et al., 2005; Bunzeck et al., 2010; Park et al., 2012; Diederen et al., 2017). During this second phase (i.e., adaptive task), three visual cues (60 triangles, 60 squares; 60 diamonds; see Figure 1) were randomly intermixed and presented in central vision for $1500 \mathrm{~ms}$ on a gray background. Each cue was followed by a fixation cross that was shown throughout the remaining trial. Three-thousand millisecond after the offset of the cue, an electrical stimulation was applied for $500 \mathrm{~ms}$. The three types of cues were associated with different contexts and predicted the occurrence of an electrical stimulation with either: (1) a high or medium; (2) a medium or low; and (3) a high or low stimulation intensity, whereby both stimulation intensities had the same occurrence probability in each type of context (e.g., in context 2, $50 \%$ of the stimulations had a medium and $50 \%$ a low intensity). The fixation cross disappeared $4000 \pm 100 \mathrm{~ms}$ after stimulation offset. Note, that this paradigm is an event-related design.

On the first day (see above), all volunteers explicitly learned the association between the type of visual cue and stimulation magnitude in the behavioral lab outside of the scanner. The task was to judge whether the relatively low or high electrical stimulation was applied by pressing one of two buttons using the right index or middle finger, respectively. The response buttons were randomized across participants. Before the actual task in the scanner on day 2 and 3, participants were familiarized again with the task in a short practice consisting of 10 trials and accuracy and speed was stressed. The adaptive task was split into four blocks of 45 trials, and took approximately $\sim 40$ min including breaks.

Approximately $5 \mathrm{~min}$ after phase two ended, participants continued with the absolute task (i.e., phase 3 ) consisting of a different trial randomization than in phase 1. Finally, structural scans were acquired for approximately $15 \mathrm{~min}$ (see below).

Note that the relatively short jitters of $100 \mathrm{~ms}$ (Figure 1) might be longer in order to significantly improve design efficiency and should be adjusted in future experiments.

\section{fMRI Data Acquisition}

The fMRI acquisition was performed on a 3-tesla system (Siemens Trio) with echo planar imaging (EPI). During functional imaging, $48 \mathrm{~T} 2^{*}$-weighted images per volume (i.e., covering whole head) with BOLD contrast were obtained (matrix, $64 \times 64 ; 48$ oblique axial slices per volume angled at $-30^{\circ}$ along the anteroposterior axis; spatial resolution: $2 \times 2 \times 2 \mathrm{~mm} ; \mathrm{TR}=2870 \mathrm{~ms}$ ). For each subject, functional MRI data were acquired for both absolute tasks each consisting of 140 volumes and the adaptive task that was split into four scanning sessions each consisting of 148 volumes per session. Six additional volumes per scanning session were recorded at the beginning of each block to allow for steady-state magnetization; these were excluded from the analyses. At the end of the experiment, anatomical images of each subject's brain were collected using multi-echo three-dimensional fast low angle shot (FLASH) acquisition for mapping $\mathrm{T} 1$ ( $\mathrm{TR}=19 \mathrm{~ms}$ ), and magnetization transfer $\left(\mathrm{TR}=24 \mathrm{~ms}\right.$ ) at $1-\mathrm{mm}^{3}$ resolution (Weiskopf and Helms, 2008; Steiger et al., 2016).

\section{fMRI Data Analysis}

All fMRI images were realigned to the first volume, unwarped, spatially normalized to the Montreal Neurology Institute space, and smoothed with a $4 \mathrm{~mm}$ Gaussian kernel using SPM12 (Ashburner et al., 2014). The fMRI time series data were high-pass filtered (cutoff $=128 \mathrm{~s}$ ) and whitened using an AR(1) model.

For each subject, we computed four first-level analyses by including each combination between pharmacological treatment 
and task (i.e., (1) placebo and adaptive task; (2) placebo and absolute task; (3) haloperidol and adaptive task; and (4) haloperidol and absolute task). First, we defined four regressors for the absolute task under placebo treatment: one regressor for each stimulation magnitude (i.e., low, medium and high), and one regressor for trials with incorrect responses (i.e., errors). Second, we computed a first-level analysis for the same task under haloperidol treatment, which included the same four types of regressors as in the former analysis. A third first-level analysis was computed for the adaptive task under placebo. Here, we defined 10 regressors: one regressor for each of the three cues (i.e., (1) high/medium; (2) medium/low; and (3) high/low); one regressor for each of the six possible stimulation outcome (i.e., (1) high stimulation in context 1; (2) medium stimulation in context 1 ; (3) medium stimulation in context 2; (4) low stimulation in context 2; (5) high stimulation in context 3; and (6) low stimulation in context 3), and one regressor for trials with incorrect responses (i.e., errors). Fourth, we computed a first-level analysis for the adaptive task under haloperidol including the same regressors as defined in the third analysis. Note that the order of the trials for the different stimulation magnitudes was fully randomized. To capture residual movement-related artifacts, six covariates were included (the three rigid-body translation and three rotations resulting from realignment) as regressors of no interest in all four models.

Two separate second-level random-effects analyses (i.e., for absolute task and adaptive task) were computed on the contrast images resulting from the four first-level analyses. In the first second-level model, the hemodynamic effects of stimulation magnitude were entered into a $2 \times 2 \times 3$ way analysis of variance (ANOVA) with the factor drug (placebo/haloperidol), time (phase 1/phase 3 ) and the factor stimulation magnitude (low/medium/high) to test for absolute pain effects and the influence of dopamine across time.

Second, a $2 \times 3 \times 2$ way ANOVA with the factor drug (placebo/haloperidol), stimulation context (context 1/context 2/context 3 ) and stimulation outcome (relatively low/relatively high) was computed to investigate adaptive pain effects and the impact of dopamine (see Figure 1). This enabled us to investigate main effects and their interactions. We were interested in brain regions associated with an adaptive coding effect irrespective of drug treatment, which was realized by contrast weights of " 1 " for all relatively high stimulations and " -1 " for all relatively low stimulations (see "Results" section).

All contrasts were initially thresholded at $p<0.001$ (uncorrected). Since we hypothesized a priori regions for the adaptive effect (i.e., insula and ACC: Leknes et al., 2013), we corrected for multiple comparisons using small volume correction (SVC; $p<0.05$, family-wise error (FWE)-correction, $k>5$ voxels). The masks for the regions of interest were defined using the WFU-Pickatlas (Maldjian et al., 2003). Otherwise, FWE was used as implemented in SPM12.

The sources of the effects were localized by overlaying the SPMs on a T1-weighted group image, which was generated by averaging all normalized T1-images, respectively (spatial resolution of $1 \times 1 \times 1 \mathrm{~mm}$ ).

\section{RESULTS}

\section{Behavioral Results: Questionnaire Regarding Side Effects}

To account for potential drug effects on subjective well-being (i.e., side effects: dry mouth, dry skin, blurred vision, lethargy, nausea, dizziness and headache), participants rated their subjective state on a 7-point Likert scale three times during the experiment (before drug administration, $2.5 \mathrm{~h}$ after drug intake and at the end of the experiment after $\sim 4.5 \mathrm{~h}$ ). Mean ratings across the seven symptoms for the three measurements and both drug treatments are displayed in Table 1 . The $2 \times 3$ ANOVA on subjective well-being (mean rating across seven side effects) with drug (placebo/haloperidol) and time (before drug intake, after $2.5 \mathrm{~h}$, at the end) revealed neither a main effect of drug nor an interaction between time and drug (all $p$ 's $>0.531)$. The analysis resulted in a significant main effect of time $\left(F_{(1.82,43.67)}=5.64\right.$; $p=0.009$ ). However, post hoc paired $t$-tests revealed no difference between the three time points when $p$ values were Bonferroni corrected (all $p$ 's $>0.113$ )

During calibration, averaged intensity ratings for the highest electrical stimulation on the VAS from 0 (i.e., electrical stimulation is not perceptible) to 10 (i.e., electrical stimulation is intolerable) did not differ between placebo $(M=1.42 \mathrm{~mA}$; $\mathrm{SD}=0.45$; range $1.42-2.3 \mathrm{~mA}$ ) and haloperidol treatment $(M=1.45 \mathrm{~mA} ; \mathrm{SD}=0.63$; range $1.44-3.00 \mathrm{~mA} ; p=0.840)$.

\section{Behavioral Results: Absolute Task}

Mean reaction times (RTs) are displayed in Table 2. A $2 \times 3 \times 2$ way ANOVA with the factor drug (placebo/ haloperidol), stimulation magnitude (low/medium/high) and time (phase $1 /$ phase 3 ) revealed a significant main effect of stimulation magnitude $\left(F_{(2,1.48)}=31.37 ; p<0.0001\right)$. Mean RTs for medium stimulation were slower than for high $\left(t_{(19)}=-3.76\right.$; Bonferroni corrected $p=0.009)$ and low stimulation $\left(t_{(19)}=8.50\right.$; Bonferroni corrected $p=0.003)$; and participants responded faster to high stimulation than to low stimulation $\left(t_{(19)}=4.17\right.$;

TABLE 1 | Likert ratings for potential side effects ( 1 = no side effects; 7 = extreme side effects) before, during and after the $\mathrm{fMRI}$ experiment in the placebo and haloperidol condition $(n=20)$.

\begin{tabular}{lccc}
\hline Treatment & $\begin{array}{c}\text { 1st assessment } \\
\text { (before DI) }\end{array}$ & $\begin{array}{c}\text { 2nd assessment } \\
\text { (2.5 h after DI) }\end{array}$ & $\begin{array}{c}\text { 3rd assessment } \\
\text { (4.5 h after DI) }\end{array}$ \\
\hline Placebo & $1.54(0.46)$ & $1.43(0.30)$ & $1.44(0.39)$ \\
Haloperidol & $1.54(0.44)$ & $1.41(0.39)$ & $1.53(0.42)$
\end{tabular}

Values represent mean ratings across-subject means (SD). DI, drug intake.

TABLE 2 | Reaction times (RTs) of hits in the absolute task for the first and third phase $(n=20)$.

\begin{tabular}{llccc}
\hline \multirow{2}{*}{ Drug } & Phase & \multicolumn{3}{c}{ Stimulation magnitude } \\
\cline { 3 - 5 } & & Low & Medium & High \\
\hline Placebo & I & $1115(193)$ & $1384(192)$ & $1259(180)$ \\
& III & $1119(206)$ & $1373(293)$ & $1191(168)$ \\
Haloperidol & I & $1111(301)$ & $1465(227)$ & $1241(377)$ \\
& III & $1135(172)$ & $1390(210)$ & $1268(257)$
\end{tabular}

Values represent RTs of correct responses across-subject means in ms (SD). 
Bonferroni corrected $p=0.009)$. Analyses showed no main effect of drug, time or an interaction between any of the factors (all p's $>0.191$ ).

The mean proportion of hits for each condition is displayed in Table 3. A $2 \times 3 \times 2$ way ANOVA on the mean hit rate revealed no main effect of stimulation magnitude, drug treatment and there was no interaction between any factors (all p's $>0.081$ ).

\section{Behavioral Results: Adaptive Task}

The mean RTs are depicted in Table 4 . A $2 \times 3 \times 2$ ANOVA with the factor drug (placebo/haloperidol), context (1/2/3) and relative stimulation outcome (low/high) revealed a significant main effect of context $\left(F_{(1.91,40.03)}=44.03 ; p<0.0001\right)$, stimulation outcome $\left(F_{(1,19)}=34.07 ; p<0.0001\right)$ and a significant interaction between context and stimulation outcome $\left(F_{(1.94,36,84)}=85.49 ; p<0.0001\right)$, but there was no effect of drug $(p$ 's $>0.214)$. Mean RTs in response to the relatively lower stimulation were faster than to the high stimulation (see Table 4). Mean RTs in context 3 were significantly faster than RTs in context 1 and context 2, where the physical difference between the stimulation is relatively smaller. Mean RTs in context 1 and 2 did not differ. Mean RTs in response to the low stimulation magnitude in both contexts (i.e., context 2: $\left.t_{(19)}=10.53 ; p<0.0001\right)$; context $3:\left(t_{(19)}=3.42\right.$; Bonferroni

TABLE 3 | Proportion of hits in the absolute task for the first and third phase $(n=20)$.

\begin{tabular}{llccc}
\hline \multirow{2}{*}{ Drug } & Phase & \multicolumn{3}{c}{ Stimulation magnitude } \\
\cline { 3 - 5 } & & Low & Medium & High \\
\hline Placebo & I & $0.83(0.11)$ & $0.70(0.14)$ & $0.72(0.21)$ \\
& III & $0.76(0.19)$ & $0.74(0.19)$ & $0.77(0.19)$ \\
Haloperidol & I & $0.74(0.24)$ & $0.66(0.15)$ & $0.64(0.22)$ \\
& III & $0.76(0.16)$ & $0.75(0.14)$ & $0.68(0.12)$ \\
\hline
\end{tabular}

Values represent proportions of correct responses across-subject means (SD).

TABLE 4 | RTS of hits in the adaptive task $(n=20)$.

\begin{tabular}{llcc}
\hline \multirow{2}{*}{ Drug } & Context & \multicolumn{2}{c}{ Relative stimulation magnitude } \\
\cline { 3 - 4 } & & Low & High \\
\hline Placebo & 1 (med/high) & $1276(243)$ & $1272(272)$ \\
& 2 (low/med) & $1104(145)$ & $1447(212)$ \\
Haloperidol & 3 (low/high) & $1041(161)$ & $1119(186)$ \\
& 1 (med/high) & $1377(243)$ & $1289(267)$ \\
& 2 (low/med) & $1127(147)$ & $1489(254)$ \\
& 3 (low/high) & $1070(219)$ & $1158(221)$ \\
\hline
\end{tabular}

Values represent RTs of correct responses across-subject means in ms (SD).

TABLE 5 | Proportion of hits in the adaptive task $(n=20)$.

\begin{tabular}{lllc}
\hline \multirow{2}{*}{ Drug } & Context & \multicolumn{2}{c}{ Relative stimulation magnitude } \\
\cline { 3 - 4 } & & \multicolumn{1}{c}{ Low } & High \\
\hline Placebo & 1 (med/high) & $0.90(0.09)$ & $0.87(0.12)$ \\
& 2 (low/med) & $0.86(0.12)$ & $0.78(0.18)$ \\
Haloperidol & 3 (low/high) & $0.96(0.06)$ & $0.96(0.08)$ \\
& 1 (med/high) & $0.86(0.12)$ & $0.87(0.10)$ \\
& 2 (low/med) & $0.87(0.10)$ & $0.75(0.17)$ \\
& 3 (low/high) & $0.93(0.10)$ & $0.93(0.08)$ \\
\hline
\end{tabular}

Values represent proportions of correct responses across-subject means (SD). corrected $p=0.009$; other $p=0.107$ ) were faster in comparison to the response to the relatively high stimulation outcome. There was no difference in mean RTs between the medium and high stimulation (i.e., context 1 ).

The proportion of correct responses is summarized in Table 5. A $2 \times 3 \times 2$ ANOVA revealed a significant main effect of context $\left(F_{(1.79,34.07)}=75.65 ; p<0.0001\right)$ and a significant interaction between context and stimulation outcome $\left(F_{(1.3425 .54)}=4.09\right.$; $p=0.043$ ), but no interaction with the factor drug (other $p$ 's $>0.089$ ). Separate post hoc $t$-tests for each context revealed no difference between both stimulation outcomes (all $p$ 's $>0.093$ ).

\section{Imaging Results for Absolute Task (Phase 1 and 3)}

Imaging data for the absolute task in phase 1 and 3 were analyzed in a $2 \times 3 \times 2$ ANOVA with the factors drug (placebo/haloperidol), stimulation magnitude (low, medium, high) and time (phase 1/phase 3). In a first step, we aimed to pinpoint brain regions that were associated with the processing of pain irrespective of drug and stimulation magnitude across both phases and drug conditions (i.e., main effect of pain, Figure 2A and https://neurovault.org/images/57884/). Whole brain analyses revealed BOLD effects in left central operculum (-54 -18 22; 24981 voxels; FWE corrected $p<0.0001)$; right putamen (14 6 - 10; 396 voxels; FWE corrected $p<0.0001)$; right angular gyrus (34-62 50; 62 voxels; FWE corrected $p=0.014)$; and cerebellar vermal lobules I-V $(4-62-12 ; 117$ voxels; FWE corrected $p<0.0001)$. Although this activation pattern is widely distributed and rather unspecific in nature, it confirms previous studies on pain processing because it involves the expected brain regions (see "Introduction" section).

In a second step, the analysis revealed BOLD responses that linearly coded absolute stimulation magnitude irrespective of drug treatment (i.e., main effect of absolute coding) in the left

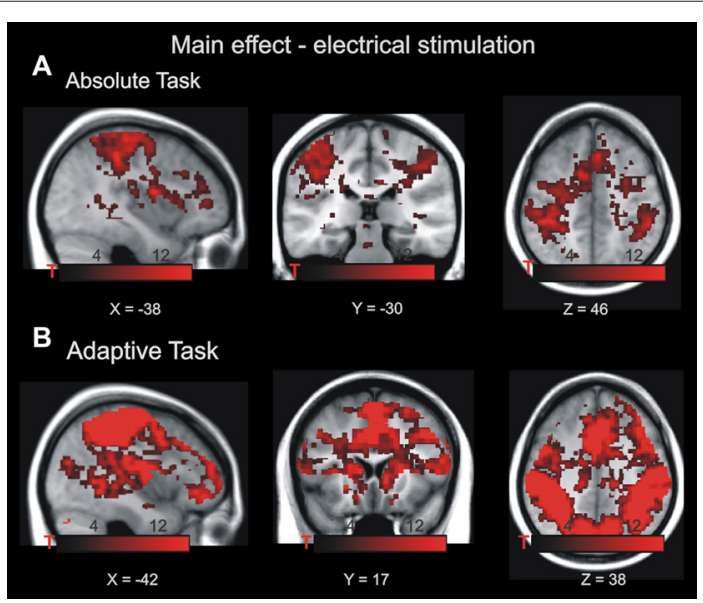

FIGURE 2 | Main effect of electrical stimulation in the absolute task (A) and adaptive task (B). The highlighted voxels exhibited increased BOLD activity during noxious stimulation $p<0.001$, family-wise error (FWE)-corrected at cluster level. Maps of activations are superimposed on a T1 group template. Note that both tasks require different cognitive demands; therefore, both main effects are not formally compared. 


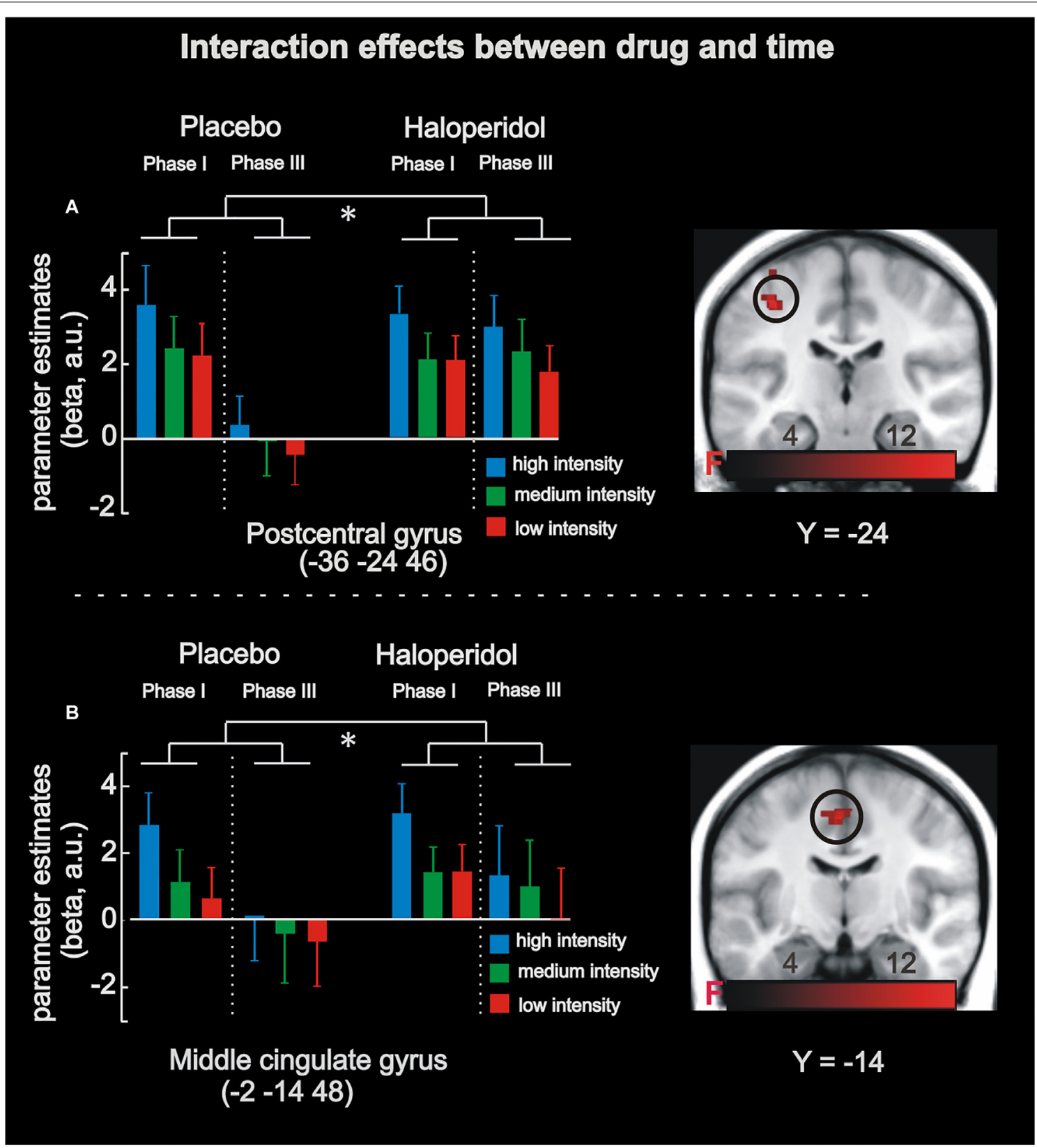

FIGURE 3 | fMRI results in the absolute task. Analyses revealed a significant interaction between stimulation magnitude, drug and time in left postcentral gyrus (A) and left midcingulate cortex (B). Under placebo in phase I, activity increased as a function of stimulation magnitude (absolute coding), and this effect was absent in phase III (i.e., habituation). Under haloperidol, however, there was no significant habituation from phase I to phase III. The significant interaction effects are highlighted by the asterisk. Maps of activations are superimposed on a T1 group template. Error-bars denote one standard error of the mean.

midcingulate cortex supplementary motor cortex $(-2-1448$; 206 voxels; FWE-corrected $p<0.0001)$ and left postcentral gyrus ( $-38-2448$; 422 voxels; FWE-corrected $p<0.0001)$. Finally, a significant $2 \times 3 \times 2$ interaction effect was observed in the left postcentral gyrus $(-36-2446 ; 123$ voxels; FWE-corrected $p<0.0001)$ and the left midcingulate cortex $(-2-1448$; 91 voxels; FWE-corrected $p<0.0001$ ), contralateral to the stimulated region. This effect was driven by a linear activity increase of stimulation magnitude in phase 1 for both drug conditions (placebo and haloperidol), and, importantly, this linear effect disappeared in phase 3 in the placebo but not haloperidol condition (see Figure 3). Thus, a time-dependent neural habituation to painful events (placebo) was blocked by haloperidol.

\section{Imaging Results for Adaptive Task}

Imaging data for the adaptive task (phase 2) were analyzed in a $3 \times 2$ ANOVA with the factors drug (placebo/haloperidol), 
context (high/medium, medium/low, high/low). First, we were interested in pain-related activity independent of context and drug (i.e., main effect of pain, Figure 2B and https://neurovault.org/images/57885/). BOLD effects were evident in bilateral postcentral gyrus $(-44-2256$; 83447 voxels; FWE-corrected $p<0.0001 ; 64-1432$; 1403 voxels; FWE-corrected $p<0.0001)$; left supplementary cortex/midcingulate cortex $(-2-650 ; 4075$ voxels; FWE-corrected $p<0.0001)$; bilateral anterior insula $(-42-66$; 582 voxels; FWE-corrected $p<0.0001 ; 44-24$; 587 voxels; FWE-corrected $p<0.0001)$. Similar to the main effect of pain in the absolute task, this activation pattern is widely distributed and rather unspecific in nature, but it confirms previous studies on pain processing (see "Introduction" section).

In the next step, we identified brain regions with adaptively coded responses to stimulation outcome irrespective of drug treatment (i.e., main effect of adaptive coding). FWE-corrected whole brain analysis did not reveal any significant effects. Subsequently, a SVC was performed, using the insula and ACC as masks (see "Introduction" section: Ploner et al., 2011; Leknes et al., 2013); this analysis also did not reveal any significant main effects or interactions $(p<0.05$, FWE-corrected, $k>5$ voxel).

\section{DISCUSSION}

\section{Summary of Main Findings}

We investigated the neural mechanisms underlying pain processing with a focus on contextual effects and dopaminergic neurotransmission in a pharmacological fMRI experiment. Our data reveal that haloperidol decreased neural adaptation to electrical stimulation in pain-associated areas (Iannetti and Mouraux, 2010), including left postcentral gyrus and left midcingulate cortex. While this habituation effect was evident under placebo, it was absent after haloperidol intake (see Figure 3) suggesting a direct link to dopaminergic neurotransmission.

\section{fMRI Findings for Absolute Task}

Absolute coding of pain magnitude in the left midcingulate cortex and left postcentral gyrus is consistent with previous fMRI studies reporting BOLD increases as a function of pain intensity in brain regions associated with pain or saliency processing. This includes primary and secondary somatosensory cortices, the insular cortex and mid/ACC (Coghill et al., 1999; Büchel et al., 2002; Iannetti and Mouraux, 2010). Specifically, the midcingulate cortex has been involved in cognitive control processes, preparation of defensive responses to threat (for review, see Shackman et al., 2011) and in discriminating between pain intensities (Büchel et al., 2002).

The habituation effect in the left postcentral gyrus and mid-cingulate cortex in the placebo group corresponds to previous research showing that habituation to nociceptive stimuli can already be evident within a short period of time (e.g., Milne et al., 1991). For instance, electro-dermal stimulation is associated with a reduction of BOLD responses from the first to the second half of the experiment in primary and secondary somatosensory cortices, the insular and anterior/mid cingulate cortex (Mobascher et al., 2010; see also Ibinson et al., 2004; Bingel et al., 2007; Christmann et al., 2007). Similar findings on habituation have been reported by Rennefeld et al. (2010) and Nickel et al. (2014). Also in line with previous literature, our effects were evident contralateral to the stimulated hand (e.g., Peyron et al., 2000; Bingel et al., 2003). However, in comparison to former habituation studies, we used three different stimulation magnitudes, which all showed habituation over time. The absence of a graded reduction of BOLD response for the three different pain magnitudes in the third phase (i.e., absence of an absolute effect) indicates that habituation to pain is rather an all-or none phenomenon.

Importantly, the interaction between drug and time provides evidence for the role of dopamine in pain processing. At the physiological level, haloperidol reduces dopamine availability by blocking dopamine D2 receptors (Kapur et al., 1997) and striatal dopamine D2 receptors are known to modulate pain processing (Hagelberg et al., 2004; Potvin et al., 2009). Thus, blocking dopaminergic neurotransmission may have prevented the habituation to electrical stimulation across time. Indeed, clinical studies suggest that habituation is reduced in chronic pain patients, such as fibromyalgia or migraine (Valeriani et al., 2003; Montoya et al., 2006; Smith et al., 2008), with a link to dopaminergic deficits (Potvin et al., 2009). For instance, Parkinson's patients showed reduced neural and behavioral habituation to pain stimuli in the absence of levodopa treatment (i.e., dopaminergic stimulation; Schestatsky et al., 2007; see also Martikainen et al., 2015). Moreover, animal models stressed that chronic pain is associated with decreased D2 receptor availability and excitatory functions of D2 neurons in the nucleus accumbens (Schwartz et al., 2014). Finally, intraventricular or striatal microinjections of haloperidol increased acute pain and apomorphine (dopamine agonist) reduced nociception (Lin et al., 1981; Magnusson and Fisher, 2000; Mansikka et al., 2005). Thus, our findings provide evidence for the role of dopamine in pain processing by showing reduced neural habituation following receptor blockage in pain-associated brain regions.

\section{No fMRI Findings for Adaptive Task}

Contrary to our predictions, adaptive coding was not evident in the mesolimbic system, including the ventral striatum (e.g., Bunzeck et al., 2010; Park et al., 2012) and dopaminergic SN/VTA (Tobler et al., 2005; Matsumoto and Hikosaka, 2009; Bauch et al., 2014; Diederen et al., 2016). One possibility for this null finding is that the spatial resolution of fMRI could have been too low to dissociate between subsets of SN/VTA neurons showing adaptive coding and others responding in an absolute fashion. Alternatively, adaptive coding within the dopaminergic system might depend on task properties. In fact, hemodynamic responses within the human SN/VTA and ventral striatum were adaptively coded in a paradigm where reward distributions alternated in short blocks (rather than trial wise) and had to be learned throughout the experiment (Diederen et al., 2016). 
This indicates that adaptive coding to appetitive and aversive information may be more pronounced in implicit and blocked learning paradigms. Together with higher spatial resolution, both aspects should be regarded in future studies. A third possibility is that adaptive coding of nociceptive events does not depend on the dopaminergic mesolimbic system but is driven by other neuromodulators such as the opioid or norepinephrine system (e.g., Wager et al., 2007; Scott et al., 2008).

For the insula and ACC, we also hypothesized adaptive coding effects on the basis of a study by Leknes et al. (2013), which could not be confirmed. Here, our null effects might be due to differences in the design (block vs. event related), or related to differences in sensory modality (heat vs. electrical stimuli). A final possibility is that adaptive coding is not as relevant to pain processing as it is for reward. Indeed, rewarding stimuli, such as monetary incentives, have a much wider range, which might require higher fidelity, while aversive stimuli on the other hand have a natural upper limit.

\section{Limitations}

There was no behavioral effect of haloperidol in any task of the combined pharmacological fMRI study. Instead, participants were equally able to differentiate between stimulation intensities irrespective of time and drug. This suggests that dopamine does not influence the perceptual and discriminative processes of nociceptive information per se, but may indirectly modulate pain processing via higher cognitive functions, such as learning or valuation processes (see also Becker et al., 2013; Tiemann et al., 2014). Since we only sampled the discriminating performance between different pain magnitudes, it is an open question whether subjective pain ratings to the different electrical stimulation also habituate over time (e.g., Bingel et al., 2007; Mobascher et al., 2010) and vary as a function of haloperidol. Alternatively, the effect of haloperidol on neural processes but not behavior may be due to the relatively low single dosage. Indeed, similar reports (i.e., no effects of drug on behavior) have been published in the field of placebo research (Wrobel et al.,

\section{REFERENCES}

Andreou, C., Moritz, S., Veith, K., Veckenstedt, R., and Naber, D. (2014). Dopaminergic modulation of probabilistic reasoning and overconfidence in errors: a double-blind study. Schizophr. Bull. 40, 558-565. doi: 10.1093/schbul/sbt064

Ashburner, J., Barnes, G., Chen, C., Daunizeau, J., Flandin, G., Friston, K., et al. (2014). SPM12 Manual The FIL Methods Group (and Honorary Members). Available online at: http://www.fil.ion.ucl.ac.uk/spm/software/spm12/

Bauch, E. M., Rausch, V. H., and Bunzeck, N. (2014). Pain anticipation recruits the mesolimbic system and differentially modulates subsequent recognition memory. Hum. Brain Mapp. 35, 4594-4606. doi: 10.1002/hbm.22497

Becker, S., Ceko, M., Louis-Foster, M., Elfassy, N. M., Leyton, M., Shir, Y., et al. (2013). Dopamine and pain sensitivity: neither sulpiride nor acute phenylalanine and tyrosine depletion have effects on thermal pain sensations in healthy volunteers. PLoS One 8:e80766. doi: 10.1371/journal.pone.0080766

Bingel, U., Quante, M., Knab, R., Bromm, B., Weiller, C., and Büchel, C. (2003). Single trial fMRI reveals significant contralateral bias in responses to laser pain within thalamus and somatosensory cortices. Neuroimage 18, 740-748. doi: 10.1016/s1053-8119(02)00033-2
2014) and pain sensitivity (D2 antagonist sulpiride, Becker et al., 2013).

As a final remark, we would like to point out that future research needs to include other non-nociceptive types of stimuli to investigate whether the dopaminergic effect on habituation is specific to pain processing.

\section{CONCLUSION}

Haloperidol changed the habituation to painful events over time in left postcentral gyrus and left midcingulate cortex. As such, our results point towards a previously unreported mechanism linking dopaminergic neuromodulation and habituation to pain in healthy humans.

\section{AUTHOR CONTRIBUTIONS}

EMB, VHR and NB designed research. EMB and CA performed fMRI data acquisition. EMB, NB, CA and VHR interpreted the data. EMB and NB drafted the article. CA and VHR revised the article critically for important intellectual content. EMB, CA, VHR and NB gave final approval for the article to be published; agreed to be accountable for all aspects of the work.

\section{FUNDING}

This work was supported by grants from the German Research Foundation (Deutsche Forschungsgemeinschaft, BU 2670/1-2) to NB and Hamburg state cluster of excellence (neurodapt!).

\section{ACKNOWLEDGMENTS}

We thank all participants for their willingness to participate in this study and Simon Pfeifer for helping with the fMRI data acquisition.
Bingel, U., Schoell, E., Herken, W., Büchel, C., and May, A. (2007). Habituation to painful stimulation involves the antinociceptive system. Pain 131, 21-30. doi: 10.1016/j.pain.2006.12.005

Brefel-Courbon, C., Payoux, P., Thalamas, C., Ory, F., Quelven, I., Chollet, F., et al. (2005). Effect of levodopa on pain threshold in Parkinson's disease: a clinical and positron emission tomography study. Mov. Disord. 20, 1557-1563. doi: $10.1002 /$ mds. 20629

Brenner, N., Bialek, W., and de Ruyter van Steveninck, R. (2000). Adaptive rescaling maximizes information transmission. Neuron 26, 695-702. doi: 10.1016/s0896-6273(00)81205-2

Bromberg-Martin, E. S., Matsumoto, M., and Hikosaka, O. (2010). Dopamine in motivational control: rewarding, aversive, and alerting. Neuron 68, 815-834. doi: 10.1016/j.neuron.2010.11.022

Brown, C. A., Seymour, B., Boyle, Y., El-Deredy, W., and Jones, A. K. P. (2008). Modulation of pain ratings by expectation and uncertainty: behavioral characteristics and anticipatory neural correlates. Pain 135, 240-250. doi: 10.1016/j.pain.2007.05.022

Büchel, C., Bornhövd, K., Quante, M., Glauche, V., Bromm, B., and Weiller, C. (2002). Dissociable neural responses related to pain intensity, stimulus intensity, and stimulus awareness within the anterior cingulate cortex: a 
parametric single-trial laser functional magnetic resonance imaging study. J. Neurosci. 22, 970-976.

Bunzeck, N., Dayan, P., Dolan, R. J., and Duzel, E. (2010). A common mechanism for adaptive scaling of reward and novelty. Hum. Brain Mapp. 31, 1380-1394. doi: 10.1002/hbm.20939

Christmann, C., Koeppe, C., Braus, D. F., Ruf, M., and Flor, H. (2007). A simultaneous EEG-fMRI study of painful electric stimulation. Neuroimage 34, 1428-1437. doi: 10.1016/j.neuroimage.2006.11.006

Coghill, R. C., Sang, C. N., Maisog, J. M., and Iadarola, M. J. (1999). Pain intensity processing within the human brain: a bilateral, distributed mechanism. J. Neurophysiol. 82, 1934-1943.

de Tommaso, M., Federici, A., Santostasi, R., Calabrese, R., Vecchio, E., Lapadula, G., et al. (2011). Laser-evoked potentials habituation in fibromyalgia. J. Pain 12, 116-124. doi: 10.1016/j.jpain.2010.06.004

Diederen, K. M. J., Spencer, T., Vestergaard, M. D., Fletcher, P. C., and Schultz, W. (2016). Adaptive prediction error coding in the human midbrain and striatum facilitates behavioral adaptation and learning efficiency. Neuron 90, 1127-1138. doi: 10.1016/j.neuron.2016.04.019

Diederen, K. M. J., Ziauddeen, H., Vestergaard, M. D., Spencer, T., Schultz, W., and Fletcher, P. C. (2017). Dopamine modulates adaptive prediction error coding in the human midbrain and striatum. J. Neurosci. 37, 1708-1720. doi: 10.1523/JNEUROSCI.1979-16.2016

Fairhall, A. L., Lewen, G. D., Bialek, W., and de Ruyter van Steveninck, R. R. (2001). Efficiency and ambiguity in an adaptive neural code. Nature 412, 787-792. doi: $10.1038 / 35090500$

Glaser, E. M., and Whittow, G. C. (1953). Evidence for a non-specific mechanism of habituation. J. Physiol. 122, 43-44.

Haaker, J., Lonsdorf, T. B., Thanellou, A., and Kalisch, R. (2013). Multimodal assessment of long-term memory recall and reinstatement in a combined cue and context fear conditioning and extinction paradigm in humans. PLoS One 8:e76179. doi: 10.1371/journal.pone.0076179

Hagelberg, N., Jääskeläinen, S. K., Martikainen, I. K., Mansikka, H., Forssell, H., Scheinin, H., et al. (2004). Striatal dopamine D2 receptors in modulation of pain in humans: a review. Eur. J. Pharmacol. 500, 187-192. doi: 10.1016/j.ejphar. 2004.07.024

Iannetti, G. D., and Mouraux, A. (2010). From the neuromatrix to the pain matrix (and back). Exp. Brain Res. 205, 1-12. doi: 10.1007/s00221-0102340-1

Ibinson, J. W., Small, R. H., Algaze, A., Roberts, C. J., Clark, D. L., and Schmalbrock, P. (2004). Functional magnetic resonance imaging studies of painan investigation of signal decay during and across sessions. Anesthesiology 101, 960-969. doi: 10.1097/00000542-200410000-00022

Jarcho, J. M., Mayer, E. A., Jiang, Z. K., Feier, N. A., and London, E. D. (2012). Pain, affective symptoms, and cognitive deficits in patients with cerebral dopamine dysfunction. Pain 153, 744-754. doi: 10.1016/j.pain.2012.01.002

Kapur, S., Zipursky, R., Roy, P., Jones, C., Remington, G., Reed, K., et al. (1997). The relationship between D2 receptor occupancy and plasma levels on low dose oral haloperidol: a PET study. Psychopharmacology 131, 148-152. doi: $10.1007 /$ s002130050277

Kobayashi, S., Pinto de Carvalho, O., and Schultz, W. (2010). Adaptation of reward sensitivity in orbitofrontal neurons. J. Neurosci. 30, 534-544. doi: 10.1523/JNEUROSCI.4009-09.2010

Leknes, S., Berna, C., Lee, M. C., Snyder, G. D., Biele, G., and Tracey, I. (2013). The importance of context: when relative relief renders pain pleasant. Pain 154, 402-410. doi: 10.1016/j.pain.2012.11.018

Lin, M. T., Wu, J. J., Chandra, A., and Tsay, B. L. (1981). Activation of striatal dopamine receptors induces pain inhibition in rats. J. Neural Transm. 51, 213-222. doi: 10.1007/bf01248953

Lonsdorf, T. B., Haaker, J., and Kalisch, R. (2014). Long-term expression of human contextual fear and extinction memories involves amygdala, hippocampus and ventromedial prefrontal cortex: a reinstatement study in two independent samples. Soc. Cogn. Affect. Neurosci. 9, 1973-1983. doi: 10.1093/scan/ nsu018

Magnusson, J. E., and Fisher, K. (2000). The involvement of dopamine in nociception: the role of $\mathrm{D}_{1}$ and $\mathrm{D}_{2}$ receptors in the dorsolateral striatum. Brain Res. 855, 260-266. doi: 10.1016/s0006-8993(99)02396-3

Maldjian, J. A., Laurienti, P. J., Kraft, R. A., and Burdette, J. H. (2003). An automated method for neuroanatomic and cytoarchitectonic atlas-based interrogation of fMRI data sets. Neuroimage 19, 1233-1239. doi: 10.1016/s1053-8119(03)00169-1

Mansikka, H., Erbs, E., Borrelli, E., and Pertovaara, A. (2005). Influence of the dopamine D2 receptor knockout on pain-related behavior in the mouse. Brain Res. 1052, 82-87. doi: 10.1016/j.brainres.2005.06.021

Martikainen, I. K., Nuechterlein, E. B., Peciña, M., Love, T. M., Cummiford, C. M., Green, C. R., et al. (2015). Chronic back pain is associated with alterations in Dopamine neurotransmission in the ventral striatum. J. Neurosci. 35, 9957-9965. doi: 10.1523/JNEUROSCI.4605-14.2015

Matsumoto, M., and Hikosaka, O. (2009). Two types of dopamine neuron distinctly convey positive and negative motivational signals. Nature 459, 837-841. doi: 10.1038/nature08028

May, A., Rodriguez-Raecke, R., Schulte, A., Ihle, K., Breimhorst, M., Birklein, F., et al. (2012). Within-session sensitization and between-session habituation: a robust physiological response to repetitive painful heat stimulation. Eur. J. Pain 16, 401-409. doi: 10.1002/j.1532-2149.2011.00023.x

Milne, R. J., Kay, N. E., and Irwin, R. J. (1991). Habituation to repeated painful and non-painful cutaneous stimuli: a quantitative psychophysical study. Exp. Brain Res. 87, 438-444. doi: 10.1007/bf00231861

Mobascher, A., Brinkmeyer, J., Warbrick, T., Musso, F., Schlemper, V., Wittsack, H. J., et al. (2010). Brain activation patterns underlying fast habituation to painful laser stimuli. Int. J. Psychophysiol. 75, 16-24. doi: 10.1016/j.ijpsycho.2009.10.008

Montoya, P., Sitges, C., García-Herrera, M., Rodríguez-Cotes, A., Izquierdo, R., Truyols, M., et al. (2006). Reduced brain habituation to somatosensory stimulation in patients with fibromyalgia. Arthritis Rheum. 54, 1995-2003. doi: 10.1002/art.21910

Nickel, F. T., Ott, S., Möhringer, S., Saake, M., Dörfler, A., Seifert, F., et al. (2014). Brain correlates of short-term habituation to repetitive electrical noxious stimulation. Eur. J. Pain 18, 56-66. doi: 10.1002/j.1532-2149.2013.00339.x

Nieuwenhuis, S., Heslenfeld, D. J., von Geusau, N. J., Mars, R. B., Holroyd, C. B., and Yeung, N. (2005). Activity in human reward-sensitive brain areas is strongly context dependent. Neuroimage 25, 1302-1309. doi: 10.1016/j. neuroimage.2004.12.043

Ohzawa, I., Sclar, G., and Freeman, R. D. (1982). Contrast gain control in the cat visual cortex. Nature 298, 266-268. doi: 10.1038/298266a0

Padoa-Schioppa, C. (2009). Range-adapting representation of economic value in the orbitofrontal cortex. J. Neurosci. 29, 14004-14014. doi: 10.1523/JNEUROSCI.3751-09.2009

Padoa-Schioppa, C., and Assad, J. A. (2008). The representation of economic value in the orbitofrontal cortex is invariant for changes of menu. Nat. Neurosci. 11, 95-102. doi: 10.1038/nn2020

Park, S. Q., Kahnt, T., Talmi, D., Rieskamp, J., Dolan, R. J., and Heekeren, H. R. (2012). Adaptive coding of reward prediction errors is gated by striatal coupling. Proc. Natl. Acad. Sci. U S A 109, 4285-4289. doi: 10.1073/pnas. 1119969109

Pauli, W. M., Larsen, T., Collette, S., Tyszka, J. M., Seymour, B., and O'Doherty, J. P. (2015). Distinct contributions of ventromedial and dorsolateral subregions of the human substantia nigra to appetitive and aversive learning. J. Neurosci. 35, 14220-14233. doi: 10.1523/JNEUROSCI.2277 $-15.2015$

Peters, M. L., Schmidt, A. J. M., and Van den Hout, M. A. (1989). Chronic low back pain and the reaction to repeated acute pain stimulation. Pain 39, 69-76. doi: 10.1016/0304-3959(89)90176-0

Peyron, R., Laurent, B., and García-Larrea, L. (2000). Functional imaging of brain responses to pain. A review and meta-analysis (2000). Neurophysiol. Clin. 30, 263-288. doi: 10.1016/s0987-7053(00)00227-6

Ploner, M., Lee, M. C., Wiech, K., Bingel, U., and Tracey, I. (2011). Flexible cerebral connectivity patterns subserve contextual modulations of pain. Cereb. Cortex 21, 719-726. doi: 10.1093/cercor/bhQ136

Potvin, S., Grignon, S., and Marchand, S. (2009). Human evidence of a supraspinal modulating role of dopamine on pain perception. Synapse 63, 390-402. doi: 10.1002/syn.20616

Rennefeld, C., Wiech, K., Schoell, E. D., Lorenz, J., and Bingel, U. (2010). Habituation to pain: further support for a central component. Pain 148, 503-508. doi: 10.1016/j.pain.2009.12.014

Rodriguez-Raecke, R., Ihle, K., Ritter, C., Muhtz, C., Otte, C., and May, A. (2014). Neuronal differences between chronic low back pain and depression regarding 
long-term habituation to pain. Eur. J. Pain 18, 701-711. doi: 10.1002/j.15322149.2013.00407.x

Schestatsky, P., Kumru, H., Valls-Solé, J., Valldeoriola, F., Marti, M. J., Tolosa, E., et al. (2007). Neurophysiologic study of central pain in patients with Parkinson disease. Neurology 69, 2162-2169. doi: 10.1212/01.WNL.0000295669.12443.d3

Schoenen, J., Wang, W., Albert, A., and Delwaide, P. J. (1995). Potentiation instead of habituation characterizes visual evoked potentials in migraine patients between attacks. Eur. J. Neurol. 2, 115-122. doi: 10.1111/j.1468-1331.1995. tb00103.x

Schwartz, N., Temkin, P., Jurado, S., Lim, B. K., Heifets, B. D., Polepalli, J. S., et al. (2014). Decreased motivation during chronic pain requires long-term depression in the nucleus accumbens. Science 345, 535-542. doi: 10.1126/science.1253994

Scott, D. J., Stohler, C. S., Egnatuk, C. M., Wang, H., Koeppe, R. A., and Zubieta, J. (2008). PLacebo and nocebo effects are defined by opposite opioid and dopaminergic responses. Arch. Gen. Psychiatry 65, 220-231. doi: 10.1001/archgenpsychiatry.2007.34

Shackman, A. J., Salomons, T. V., Slagter, H. A., Fox, A. S., Winter, J. J., and Davidson, R. J. (2011). The integration of negative affect, pain and cognitive control in the cingulate cortex. Nat. Rev. Neurosci. 12, 154-167. doi: $10.1038 / \mathrm{nrn} 2994$

Sjouwerman, R., Niehaus, J., and Lonsdorf, T. B. (2015). Contextual change after fear acquisition affects conditioned responding and the time course of extinction learning-implications for renewal research. Front. Behav. Neurosci. 9:337. doi: 10.3389/fnbeh.2015.00337

Smith, B. W., Tooley, E. M., Montague, E. Q., Robinson, A. E., Cosper, C. J., and Mullins, P. G. (2008). Habituation and sensitization to heat and cold pain in women with fibromyalgia and healthy controls. Pain 140, 420-428. doi: 10.1016/j.pain.2008.09.018

Steiger, T. K., Weiskopf, N., and Bunzeck, N. (2016). Iron level and myelin content in the ventral striatum predict memory performance in the aging brain. J. Neurosci. 36, 3552-3558. doi: 10.1523/JNEUROSCI.3617-15.2016

Tiemann, L., Heitmann, H., Schulz, E., Baumkötter, J., and Ploner, M. (2014). Dopamine precursor depletion influences pain affect rather than pain sensation. PLoS One 9:e96167. doi: 10.1371/journal.pone.0096167

Tobler, P. N., Fiorillo, C. D., and Schultz, W. (2005). Adaptive coding of reward value by dopamine neurons. Science 307, 1642-1645. doi: 10.1126/science. 1105370
Tremblay, L., and Schultz, W. (1999). Relative reward preference in primate orbitofrontal cortex. Nature 398, 704-708. doi: 10.1038/ 19525

Valeriani, M., de Tommaso, M., Restuccia, D., Le Pera, D., Guido, M., Iannetti, G. D., et al. (2003). Reduced habituation to experimental pain in migraine patients: a CO2 laser evoked potential study. Pain 105, 57-64. doi: 10.1016/s0304-3959(03)00137-4

Wager, T. D., Scott, D. J., and Zubieta, J.-K. (2007). Placebo effects on human $\mu$-opioid activity during pain. Proc. Natl. Acad. Sci. U S A 104, 11056-11061. doi: 10.1073/pnas.0702413104

Weiskopf, N., and Helms, G. (2008). "Multi-parameter mapping of the human brain at $1 \mathrm{~mm}$ resolution in less than 20 minutes," in Proceedings of the 16th Annual Meeting of ISMRM (Toronto, ON).

Wiech, K., and Tracey, I. (2009). The influence of negative emotions on pain: behavioral effects and neural mechanisms. Neuroimage 47, 987-994. doi: 10.1016/j.neuroimage.2009.05.059

Winston, J. S., Vlaev, I., Seymour, B., Chater, N., and Dolan, R. J. (2014). Relative valuation of pain in human orbitofrontal cortex. J. Neurosci. 34, 14526-14535. doi: 10.1523/JNEUROSCI.1706-14.2014

Wood, P. B., Schweinhardt, P., Jaeger, E., Dagher, A., Hakyemez, H., Rabiner, E. A., et al. (2007). Fibromyalgia patients show an abnormal dopamine response to pain. Eur. J. Neurosci. 25, 3576-3582. doi: 10.1111/j.1460-9568.2007. 05623.x

Wrobel, N., Wiech, K., Forkmann, K., Ritter, C., and Bingel, U. (2014). Haloperidol blocks dorsal striatum activity but not analgesia in a placebo paradigm. Cortex 57, 60-73. doi: 10.1016/j.cortex.2014.02.023

Conflict of Interest Statement: The authors declare that the research was conducted in the absence of any commercial or financial relationships that could be construed as a potential conflict of interest.

Copyright (C) 2017 Bauch, Andreou, Rausch and Bunzeck. This is an open-access article distributed under the terms of the Creative Commons Attribution License (CC BY). The use, distribution or reproduction in other forums is permitted, provided the original author(s) or licensor are credited and that the original publication in this journal is cited, in accordance with accepted academic practice. No use, distribution or reproduction is permitted which does not comply with these terms. 